\title{
Pavlovian conditioning of fear based upon hormonal mediation of prior aversive experience
}

\author{
JAMES T. CONCANNON, DAVID C. RICCIO, and JAMES McKELVEY \\ Kent State University, Kent, Ohio 44242
}

\begin{abstract}
A series of three experiments was conducted to determine if epinephrine administered to animals given prior shock might support learning to new environmental cues paired with the epinephrine injection. Experiment 1, utilizing nonnaive rats, provided results showing that such learning is possible. The effect, while dose-dependent, did not appear to be based on either epinephrine-induced place aversion or on sensitization. The results of Experiment 2 confirmed the basic finding when naive animals were utilized. Experiment 3 examined more carefully the potential contribution of generalized fear, impairment of extinction of fear, and nonspecific sensitization to the phenomenon. Since the findings did not support these alternative interpretations, it appears that epinephrine administration to previously stressed rats does indeed support new learning. The epinephrine-cue association may be mediated by either of two mechanisms: (1) higher order conditioning or (2) memory reactivation. The redintegrative function of epinephrine injections, in terms of modulating memory processing, is also discussed.
\end{abstract}

There is increasing evidence that stress-related hormones can influence learning or memory processes in a variety of ways. For example, a series of recent studies by McGaugh, Gold, and their associates (Gold \& Van Buskirk, 1975, 1976; McGaugh, Gold, Van Buskirk, \& Haycock, 1975) have shown that administration of either adrenocorticotropic hormone $(\mathrm{ACTH})$ or epinephrine following passive avoidance training can modulate the strength of the target memory. Another line of evidence implicating hormonal agents in memory comes from research on reactivating previously acquired responses. An important study in this area demonstrated that the decline in active avoidance which occurs at intermediate intervals after training (i.e., the "Kamin effect") could be prevented by exogenous administration of ACTH (Klein, 1972). Similarly, the retention deficit produced by electroconvulsive shock (ECS) or hypothermia can be alleviated by pretest injection of ACTH (Keyes, 1974; Mactutus, Smith, \& Riccio, in press) One interpretation of these studies is that the hormones produced the attributes critical for memory retrieval by reestablishing many of the internal sequalae generated during earlier fear training. Recently, Haroutunian and Riccio (1977) utilized this strategy of mimicking the internal

This research was supported by a NIH postdoctoral fellowship awarded to the first author (MH-07358) and by NIMH Grant MH 30223 to the second author. A preliminary report of this research was presented at the 1978 Psychonomic Society meetings. We thank Gary David and Deborah Ligore for their assistance in conducting the experiments. Requests for reprints should be addressed to David C. Riccio, Department of Psychology, Kent State University, Kent, Ohio 44242. stress state to reduce the retention loss typically obtained in young rats. Using a variation of Campbell and Jaynes (1966) reinstatement technique, they found that interpolating a single exposure to Pavlovian fear cues in conjunction with an injection of epinephrine or ACTH in the retention interval significantly enhanced subsequent test performance. Presumably, the cue/drug episode provided a salient reminder somewhat comparable to re-pairing of the CS and UCS.

In addition to these studies, which emphasize the UCS-like characteristics of hormones, other work demonstrates that hormonal states can function much like external cues in directing responding. Thus, epinephrine can act as a conditioned stimulus (CS) for leg flexion when paired with shock in dogs (Cook, Davidson, Davis, \& Kelleher, 1960) or serve as a discriminative stimulus $(\mathrm{S}+$ ) controlling operant responding for food in monkeys (Schuster \& Brady, 1964). Also, recent evidence suggests that exogenously administered ACTH can act as an agent which yields state-dependent learning (Gray, 1975).

One question that has received little attention is whether hormonal states can redintegrate stress experiences in a way that will support new associative learning. Since endogenous release of epinephrine occurs during a traumatic episode, the hormone should also provide a stimulus context overlapping the fear state. A subsequent exogenous administration of epinephrine might then be expected to reinduce the internal changes associated with fear. If this hormonal-based reactivation should occur in the presence of distinctive but innocuous cues, then these latter stimuli also might acquire the property of eliciting fear. 


\section{EXPERIMENT 1}

The possibility that a hormonal state associated with earlier stress can subsequently provide the basis for fear conditioning is suggested by certain findings reported by Anderson, Crowell, Koehn, and Lupo (1976). These authors found that noncontingent experience with severe shock exerted a proactive influence which reduced activity in the open-field situation 1 week later. The reduced activity seen in preshocked rats was not based upon stimulus generalization between the shock and test situations. Accordingly, the authors proposed that the internal consequences of the emotional reaction to shock became associated (as cues) with the fear response and that subsequently the mild emotional response to the novelty of the open field served (via feedback) as a CS to elicit a more pronounced fear response.

The aim of Experiment 1 was to provide a more direct evaluation of hormonal mediation of fear conditioning. The general strategy was to induce a state of arousal and mild distress in rats by presenting a series of noncontingent footshocks. Subsequently, we administered an injection of epinephrine in order to re-arouse the shock-induced state, and the animals were placed in a distinct environment (CS). Our expectation was that, when tested later for fear to the situational CS, these subjects would show greater avoidance than would appropriate control groups. It was further predicted that the magnitude of the fear response would be dose-dependent.

\section{Method}

Subjects. Thirty-two adult male Holtzman rats (360-560 g) served as subjects. These animals had served in previous experiments in which most had received a single passive avoidance training trial followed immediately by hypothermic deep-body cooling $\left(21^{\circ} \mathrm{C}\right)$. All animals were housed in gang cages of six animals or less, with food and water available ad lib. The colony in which the animals lived was maintained on a light-dark cycle of $16 \mathrm{~h} \mathrm{light} / 8 \mathrm{~h}$ dark, with the room temperature at $20^{\circ}-22^{\circ} \mathrm{C}$.

Apparatus. The apparatus for administering noncontingent footshock consisted of an unpainted pinewood box, $18.5 \mathrm{~cm}$ long $\times$ $15.5 \mathrm{~cm}$ wide $\times 20.2 \mathrm{~cm}$ high, with a clear Plexiglas lid. This box rested upon stainless steel grids that were $.2 \mathrm{~cm}$ in diameter and spaced $1.0 \mathrm{~cm}$ center-to-center in a room well illuminated by the ceiling lights. These grids were connected to a matched-impedance shock source (Campbell \& Teghtsoonian, 1958) through a Foringer scrambler (SC-901). The shock circuit was calibrated to deliver a $150-\mathrm{V} 1.0-\mathrm{sec}$ shock when triggered by a manual switch. All electromechanical programming equipment was located in a room adjacent to the NCFS apparatus.

The cue-exposure and testing apparatus consisted of a twocompartment, black-white chamber, $36.8 \times 16.7 \times 20.5 \mathrm{~cm}$, constructed of painted Plexiglas. The compartments were separated by a $.6-\mathrm{cm}$ partition with a $6.0 \times 7.7 \mathrm{~cm}$ opening which could be blocked by lowering a door. The floor of the shuttlebox consisted of stainless steel rods that were $.2 \mathrm{~cm}$ in diameter and spaced $1.0 \mathrm{~cm}$ center-to-center. A single $15-\mathrm{W}$ lightbulb, suspended $40 \mathrm{~cm}$ above the center of the white compartment, provided the only source of illumination for the white side of the box through its clear Plexiglas lid. In some cases, the clear Plexiglascovered pinewood box was set upon these grids for "neutral" cue exposure. A mirror suspended above the white chamber allowed the experimenter to continuously observe the animal.

Procedure. All animals were handled daily for $5 \mathrm{~min}$ per day for 2 days prior to the first NCFS session. The first NCFS was delivered to the animals on the day after preliminary handling. Randomly selected animals were placed in the pinewood box apparatus located in a well-illuminated experimental room. Ten seconds later, the animal received the first $150 \mathrm{~V}, 1.0-\mathrm{sec}$ footshock. Two additional shocks were delivered 30 and $50 \mathrm{sec}$ after placement in the apparatus. The animal was removed from the NCFS box $10 \mathrm{sec}$ after the last shock. This procedure was repeated $24 \mathrm{~h}$ later, so that each animal received a total of six shocks on the 2-day shock-exposure period.

The injection-plus-cue exposure phase began $24 \mathrm{~h}$ after the last NCFS session. Each of the 32 NCFS animals was randomly assigned to one of four experimental groups $(n=8$ /group). These four groups differed only in the type of injection they received prior to cue exposure. That is, separate groups of animals received a subcutaneous (nape of the neck) injection of either $.9 \%$ physiological saline, or $.01, .05$, or $.10 \mathrm{mg} / \mathrm{kg}$ epinephrine (dissolved in physiological saline) in a constant volume of $1.0 \mathrm{ml} /$ $\mathrm{kg}$. These injections were administered in a laboratory room different from that used for any other phase of the experiment. Twenty minutes later, all animals were placed into the black compartment of the exposure apparatus for a single 60 -sec cue exposure session (no shock present).

The testing procedure followed injection-plus-cue exposure by $24 \mathrm{~h}$. The test was a simple spatial (passive) avoidance test where the rat's tendency to avoid the black side of the shuttlebox was measured. Each animal was placed in the white side of the shuttlebox, facing away from the door, $10 \mathrm{sec}$ prior to the opening of the door. The tendency to avoid the black cues was measured in a single 30-min retention test, and the main dependent variable was latency to enter the black side of the apparatus.

In general, the results shown for crossover latencies also held for the total time spent avoiding black cues in the 30 -min session. However, results were most consistent across all experiments for crossover latencies; therefore, we report only crossover latencies. Shock was not delivered at any time during testing.

\section{Results and Discussion}

Figure 1 shows the mean crossover latencies at testing as a function of hormone dosage during

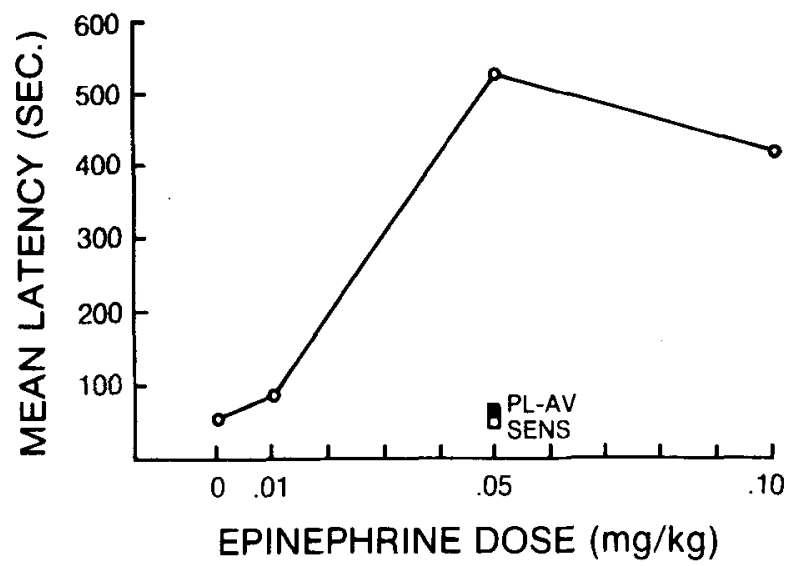

Figure 1. Mean latency of crossover response as a function of epinephrine dose for animals given prior shock and black-cue exposure (open circles). Place-aversion (PL-AV) controls received equivalent handling, apparatus exposure, and cue exposure after administration of $.05 \mathrm{mg} / \mathrm{kg}$, although no shocks were given. Sensitization (SENS) controls received treatment equivalent to experimental groups (open circles), with the exception that they were exposed to the unpainted pinewood box after $.05-\mathrm{mg} / \mathrm{kg}$ epinephrine injection. 
cue exposure. Epinephrine doses of .01 and $.05 \mathrm{mg}$ / $\mathrm{kg}$ led to a dose-dependent elevation of crossover latencies relative to the saline-injected group. The $.10-\mathrm{mg} / \mathrm{kg}$ group, while apparently excelling the saline group in its avoidance, performed at a level somewhat lower than the $.05-\mathrm{mg} / \mathrm{kg}$ group.

A one-way analysis of variance (ANOVA) of the mean crossover latencies indicated a reliable overall effect of Drug Dose $[F(3,28)=3.81, p=.02]$. Subsequent Duncan's multiple range tests (alpha $=$ .05 ) showed that the $.05-\mathrm{mg} / \mathrm{kg}$-dose group differed from all other groups except the $.10-\mathrm{mg} / \mathrm{kg}$ group, and that no other between-group differences were reliable. Hence, $.05 \mathrm{mg} / \mathrm{kg}$ epinephrine injected into rats with prior noncontingent shock experience increased their tendency to passively avoid new environmental cues paired with the hormonal state.

As a check on the possibility that avoidance of the black compartment was due to place aversion or sensitization produced by epinephrine per se, rather than to an associative process, we subsequently tested two groups of similar subjects that received either: (1) drug injection $(.05 \mathrm{mg} / \mathrm{kg})$ and cue exposure without prior NCFS (place aversion control) or (2) prior NCFS treatment followed by hormone injection and exposure to a "neutral" (pinewood box) cue (sensitization control). Mean crossover latencies for these groups are also depicted in Figure 1. Although direct statistical comparison of these groups with conditions run earlier is not appropriate, the total lack of passive avoidance suggests that epinephrine-induced place aversion, or sensitization, is not responsible for the elevated latencies of the experimental groups.

\section{EXPERIMENT 2}

Experiment 1 provides suggestive evidence that exogenous epinephrine can reinstate the animal's internal emotional reaction to prior footshocks in a manner that supports associative conditioning to the cues with which the state is now paired. However, the ad hoc nature of two of the control conditions was less than ideal; furthermore, no assessment was obtained of the effect of epinephrine-plus-cue exposure in animals which receive equivalent handling and exposure to the NCFS apparatus without any prior NCFS. Accordingly, Experiment 2 incorporated control groups that received either the hormone injection plus black cue exposure (place aversion control) or epinephrine injection plus exposure to neutral cues of a pinewood chamber. The latter served as another type of control condition for general sensitization effects of the hormone injection. In addition, because the results of Experiment 1 were obtained with nonnaive rats, which may have re- stricted generality, the present study employed experimentally naive animals.

\section{Method}

Subjects. Thirty-two naive adult male Holtzman rats (310-417 g) served as subjects. All animals were earpunched for identification and housed in single cages at least 2 days prior to preliminary handling. All other aspects of animal care and handling were identical to those used in Experiment 1.

Apparatus. The apparatus was the same as that used in Experiment 1.

Procedure. The 32 animals were randomly assigned to four experimental groups (8/group). Two groups (SAL/CUES and $\mathrm{EPI} / \mathrm{CUES}$ ) received the NCFS regimen already described in Experiment 1, followed $24 \mathrm{~h}$ later by either a saline injection or an injection of $.05 \mathrm{mg} / \mathrm{kg}$ epinephrine, respectively, and 60 -sec black-cue exposure. A third group, which served as a control for place aversion (PL-AV), was placed in the NCFS apparatus on Days 1 and 2, but no shocks were administered at this time. This group received an epinephrine injection plus cue exposure identical to that received by the first two groups. Finally, a sensitization control (SENS) condition was included which received NCFS apparatus exposure without shock on Days 1 and 2. On Day 3, this group received an epinephrine injection plus $60-\mathrm{sec}$ exposure to the clear Plexiglas-covered pine box, which was placed on the grids on which the shuttlebox was usually placed. All animals were tested on Day 4 in a fashion identical to that in Experiment 1, with original crossover latency being the main dependent variable of interest.

\section{Results and Discussion}

Figure 2 presents the mean crossover latencies for the four groups. Prior noncontingent shock in combination with later epinephrine and black cue exposure again led to reliable avoidance of the black cues relative to the animals receiving prior NCFS and a saline injection followed by cue exposure. As in Experiment 1, we did not find reliable aversion in the place aversion control group. Furthermore, animals given no prior shock but epinephrine plus pine-box cue exposure did not show reliable avoid-

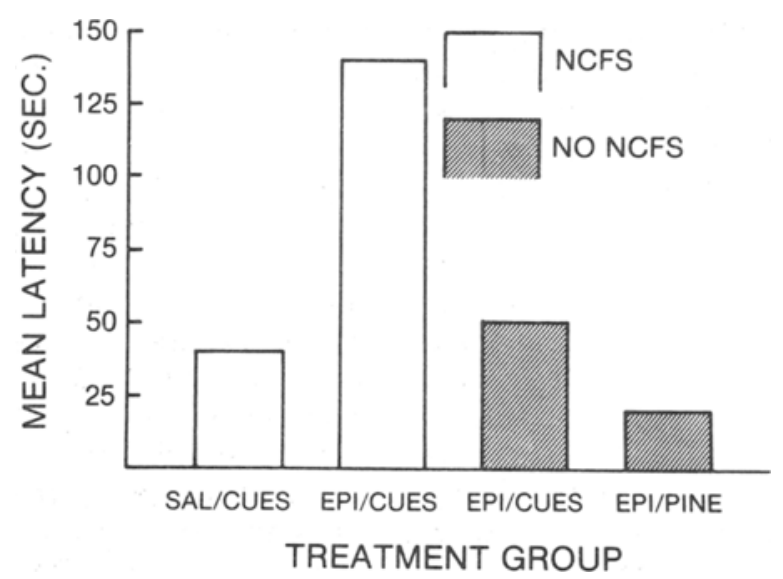

Figure 2. Mean latency of crossover response for animals receiving prior NCFS (open bars) or no prior NCFS (shaded bars). "Cues," in this case, refers to exposure to the black compartment; "pine" refers to exposure to the unpainted pinewood box cues. $(\mathrm{SAL}=$ saline; EPI $=.05 \mathrm{mg} / \mathrm{kg}$ epinephrine. $)$ 
ance. Hence, only the group given prior NCFS, epinephrine, and black-cue exposure showed reliable avoidance in this experiment.

This pattern of results was confirmed statistically using a one-way ANOVA of the crossover latencies, which revealed a reliable overall effect of Treatment Group $[F(3,28)=3.47, p<.05]$. A subsequent Duncan's multiple range test showed that Group EPI/CUES differed reliably from all other groups, while there were no other reliable between-group differences. These results replicate the general results of Experiment 1 and suggest that epinephrine can mediate a specific association between prior NCFS and black cues, perhaps by epinephrine's elicitation of a state similar in its attributes to prior NCFS. Although the difference between Groups EPI/CUES and SAL/CUES was not as substantial as in Experiment 1 , this may reflect the differences in the experiential histories of the two sets of subjects.

\section{EXPERIMENT 3}

The first two experiments suggest that epinephrine administration may re-arouse a sufficient portion of a previous noncontingent shock experience to allow the emotional state to become associated with concurrent environmental cues (i.e., the black chamber). Although there was no evidence that the effect was mediated via simple stimulus generalization from grid cues (saline controls, Experiments 1 and 2) or via place aversion from the exogenous epinephrine per se (place aversion controls), it remains possible that a form of sensitization may have contributed to the aversion. That is, perhaps prior NCFS and later hormone treatment in any context would result in heightened latencies at testing. To evaluate this possibility, Experiment 3 included a condition identical to the main experimental manipulation, except that following epinephrine injection the subjects were exposed to neutral cues rather than the CS. In addition, for half the subjects in this control condition and in the experimental condition, the usual grid floor was covered by a metal plate during the postepinephrine exposure. If sensitization were the basis for our findings, one might expect all four of these groups to show comparable aversion at testing.

A quite different potential criticism of the findings is based upon the possibility that differential extinction of immobilization or freezing elicited by the grid floor would elevate cross-through latencies in the critical hormone-cue exposure condition. While the scores in the saline control groups appear to provide evidence against the view that fear generalized directly from the NCFS apparatus to the test situations, it could be argued that in this condition the cue exposure provided an opportunity for extinction of fear elicited by the grid floor. If one further assumes that the exogenous epinephrine serves to hinder this extinction (cf. Cunningham, 1979), then the phenomenon need not represent a new associative connection between cues and internal states. The effect could be attributed to a lack of extinction to generalized fear (apparatus) cues in the hormoneinjected group. This interpretation suggests that epinephrine-injected subjects not given the specific cue exposure should perform as well as those placed in the black chamber. Although the injected groups that received exposure to a different apparatus in this experiment should bear on this hypothesis, we decided also to include a condition in which there was no apparatus exposure following epinephrine administration. Rats in this group were simply returned to their home cages at the appropriate time following the drug injection.

\section{Method}

Subjects. Forty-eight naive adult male Holtzman rats (329$498 \mathrm{~g}$ ) served as subjects. All aspects of animal identification, care and handling were identical to those used in Experiment 2.

Apparatus. The apparatus was quite similar to that used in Experiments 1 and 2. Due to unavailability of the original shuttlebox, however, the cue-exposure and testing apparatus used in this experiment consisted of a two-compartment, blackwhite chamber, $47.0 \times 17.6 \times 22.7 \mathrm{~cm}$, constructed of painted Plexiglas, with a clear Plexiglas top above the white chamber. The compartments were separated by a $.6-\mathrm{cm}$ partition with a $7.8 \times 7.8 \mathrm{~cm}$ opening blocked by lowering a door. The grid floor of the apparatus was the same as that used in Experiments 1 and 2, as was the NCFS apparatus and pine-cue box.

Procedure. The 48 animals were assigned randomly to six experimental groups ( $\mathrm{n}=8 /$ group). All animals in all conditions received the NCFS regimen described in Experiment 1 and were injected $24 \mathrm{~h}$ later with either saline or epinephrine $(.05 \mathrm{mg} / \mathrm{kg})$. Four of the groups were administered an epinephrine injection 20 min prior to the 60 -sec cue exposure, which consisted of a factorial combination of black cues vs. pine cues (described in Experiments 1 and 2) and uncovered vs. plate-covered grids. Hence, these groups were labeled: EPI/CUES/GRIDS, EPI/ CUES/PLATE, EPI/PINE/GRIDS, and EPI/PINE/PLATE, respectively, where cues vs. pine refers to the apparatus construction material for the exposure phase and GRIDS vs. PLATE refers to whether or not the grids in the exposure apparatus were covered with an aluminum plate. The fifth group (EPI/ HOME) received an epinephrine injection, was placed in the holding cage for $20 \mathrm{~min}$, and was then returned to the home cage. For a sixth group (SAL/CUES/GRIDS), saline was injected $20 \mathrm{~min}$ prior to the 60 -sec black-cue exposure, as in Experiments 1 and 2. Testing occurred for all animals on Day 4, and was conducted in a fashion identical to that described for the first two experiments.

\section{Results and Discussion}

Figure 3 presents the mean crossover latencies for the six groups in Experiment 3. Prior NCFS led to reliable avoidance of the black cues only if the animals received the black cue exposure on the uncovered grids. As in Experiments 1 and 2, Group EPI/CUES/GRIDS excelled Group SAL/CUES/ GRIDS and, as suggested in Experiment 1, Group EPI/CUES/GRIDS excelled Group EPI/PINE/ GRIDS (the "sensitization" control group). In fact, Group EPI/CUES/GRIDS excelled all other com- 


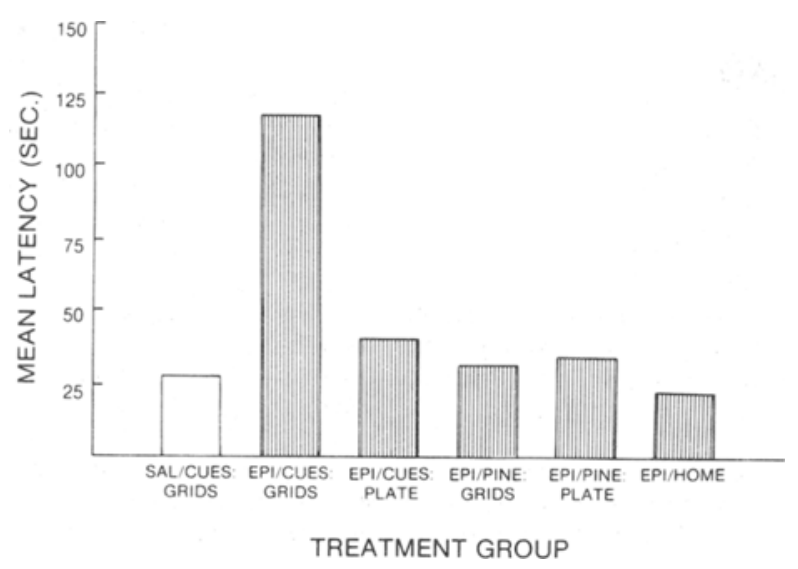

Figure 3. Mean latency of crossover response as a function of treatment group in Experiment 3. "Cues" refers to exposure to the black compartment; "Pine" refers to exposure to the unpainted pinewood-box cues. "Grids" refers to the fact that the grids in the exposure apparatus were uncovered; "plate" means that these grids were covered with a metal plate. (SAL = saline; $\mathrm{EPI}=.05 \mathrm{mg} / \mathrm{kg}$ epinephrine.)

parison groups, as suggested by our "new learning", interpretation.

This pattern of results was confirmed statistically by a one-way ANOVA of the crossover latencies, which revealed a reliable overall effect of Treatment Group $[F(5,42)=4.39, p<.003]$. A subsequent Duncan's multiple range test revealed that Group EPI/CUES/GRIDS differed reliably from all other groups, while there were no other reliable betweengroup differences. These results confirm the results of Experiments 1 and 2 and suggest that epinephrine can mediate a specific association between prior NCFS and black cues, and that this effect is not easily explained on the basis of factors such as sensitization, generalization of fear, and/or differential extinction of freezing.

\section{GENERAL DISCUSSION}

The results of three experiments demonstrate that following an episode of aversive stimulation, exogenous administration of epinephrine coupled with exposure to environmental cues results in these stimuli acquiring fear-eliciting properties. Control manipulations showed that the intermediate phase (and type) of cue exposure was critical. Other control manipulations provided no evidence that the results were attributable to (1) generalized fear from noncontingent footshock, (2) epinephrine-induced place aversion, (3) impairment of generalized fear extinction from NCFS treatment during cue exposure, or (4) a nonspecific sensitization effect. Thus, the effect appears to be based on a truly associative relationship rather than performance changes produced by separate components of the treatment. To the best of our knowledge, then, the present data provide the first demonstration that learning can be achieved by hormonal mediation of a previous state of stress.

The processes by which learning was established in this paradigm remain to be specified. A possible associative mechanism would be higher order conditioning, in which the first-order stimulus $\left(\mathrm{CS}_{1}\right.$ : epinephrine) serves to establish a CR to another stimulus $\left(\mathrm{CS}_{2}\right.$ : black cues). Presumably, during initial aversive treatment, endogenous epinephrine is rapidly released and comes to serve as an internal cue $\left(C_{1}\right)$ by virtue of its pairing with subsequent shocks. Subsequent exogenous administration of epinephrine $\left(\mathrm{CS}_{1}\right)$ might then function as an elicitor of fear capable of being associated with an environmental stimulus $\left(\mathrm{CS}_{2}\right.$ : black cues), which would result in the transfer of control of fear to these black cues. The unique feature of the present phenomenon is that a hormonal state (with associated feedback) provides the putative $\mathrm{CS}_{1}$.

Whether the present paradigm has more than a formal similarity to higher order conditioning is not yet clear. Indeed, at least two aspects of the hormoneinduced effect appear to differ from effective secondorder exteroceptive conditioning procedures: (1) the presumed $\mathrm{CS}_{1}$ (epinephrine state) probably exists to some extent prior to and following $\mathrm{CS}_{2}$ exposure, and (2) only a single "trial" was employed. However, given the difficulty of "equating" manipulations between exteroceptive and interoceptive paradigms, it may be premature to view these differences as evidence against mediation through higher order conditioning.

A related interpretation which emphasizes the role of memorial events is that the epinephrine treatment "redintegrates" the earlier state of distress and that this internal state becomes linked with contempory cues. Thus, the drug would serve as a surrogate UCS for previously shocked subjects. Conversely, epinephrine in the absence of prior shock or prior shock not followed by the epinephrine plus target cue exposure should be ineffective, as was the case. A similar hypothesis is that epinephrine administration might reactivate a memorial representation of the shock UCS (cf. Rescorla \& Heth, 1975) by virtue of their shared attributes along a stress dimension. In this case, epinephrine would not be acting as a surrogate UCS, but rather would be acting as a memorial attribute sufficiently strong to elicit a reexperience or re-presentation of the prior shock experience. This account is in accord with the ability of external agents, such as UCSs alone (Miller \& Springer, 1973; Spear \& Parsons, 1976) or administration of hormones (Klein, 1972) to alleviate a number of types of retention failure in more traditional memory paradigms. Successful alleviation 
of these retention failures is probably at least partially attributable to reinstating some memory attribute(s) related to a weakened UCS representation (Rescorla \& Heth, 1975).

One interesting implication of the phenomenon concerns the epinephrine-induced reinstatement of memory recently reported by Haroutunian and Riccio (1977). Although their study differed in numerous important respects from the present paradigm, it is possible that the improved retention of fear produced when immature animals received epinephrine in conjunction with a "reminder" exposure to the CS was based upon new learning rather than explicit memory reactivation. A comparable reinstatement group receiving NCFS in lieu of training would seem to be needed to clarify this issue.

Boundary conditions for the hormonal induced transfer of fear have not yet been established, but variables likely to influence the degree of aversion include (1) frequency and intensity of noncontingent shock experience, (2) dose of epinephrine, and (3) time between injection and exposure to the target cues. The relationships may not always be direct, however. For example, prior exposure to intense noncontingent footshock, while producing marked internal changes, may also increase generalized fear to the test conditions, thereby masking the influence of any hormonally mediated new learning. Moreover, other agents may prove more effective than epinephrine. While precise comparisons are not warranted, pilot work in our laboratory suggests that ACTH administration may produce a more substantial effect. In any event, we think the present paradigm may prove useful in exploring further a relatively neglected aspect of the relationship between endocrines and learning and memory processes.

\section{REFERENCES}

Anderson, D. C., Crowell, C., Koehn, D., \& Lupo, J. V. Different intensities of unsignalled inescapable shock treatments as determinants of non-shock-motivated open field behavior: A resolution of disparate results. Physiology \& Behavior, 1976, 17, 391-394.

Campbell, B. A., \& Jaynes, J. Reinstatement. Psychological Review, 1966, 73, 478-480.

Campbele, B. A., \& Teghtsoonian, R. Electrical and behavioral effects of different types of shock stimuli on the rat.
Journal of Comparative and Physiological Psychology, 1958, 51, 185-192.

Cook, L., Davidson, A., Davis, D. J., \& Kelleher, R. T. Epinephrine, norepinephrine and acetylcholine as conditioned stimuli for avoidance behavior. Science, 1960, 131, 990-992.

Cunningham, C. L. Alcohol as a cue for extinction: State dependency produced by conditioned inhibition. Animal Learning \& Behavior, 1979, 7, 45-52.

Gold, P. E., \& VAN BuskiRk, R. B. Facilitation of timedependent memory processes with posttrial epinephrine injections. Behavioral Biology, 1975, 13, 145-153.

Gold, P. E., \& VAN Buskink, R. Effects of posttrial hormone injections on memory processes. Hormones and Behavior, 1976, 7, 509-517.

Gray, P. Effect of adrenocorticotropic hormone on conditioned avoidance in rats interpreted as state-dependent learning. Journal of Comparative and Physiological Psychology, 1975, 88, 281-284.

Haroutunian, V., \& Riccio, D. C. Effect of arousal conditions during reinstatement treatment upon learned fear in young rats. Developmental Psychobiology, 1977, 10, 25-32.

Haroutunian, V., \& Riccio, D. C. Drug-induced "arousal" and the effectiveness of CS exposure in the reinstatement of memory. Behavioral and Neural Biology, 1979, 26, 115-120.

KEYES, J. B. Effect of ACTH on ECS-produced amnesia of a passive avoidance task. Physiological Psychology, 1974, 2, 307-309.

KLEIN, S. B. Adrenal-pituitary influence in reactivation of avoidance-learning memory in the rat after intermediate intervals. Journal of Comparative and Physiological Psychology, 1972, 79, 341-359.

Mactutus, C. F., Smith, R. L., \& Riccio, D. C. Extending the duration of ACTH-induced memory reactivation in an amnestic paradigm. Physiology \& Behavior, in press.

McGaugh, J. L., Gold, P. E., Van Buskirk, R., \& Haycock, J. Modulating influences of hormones and catecholamines on memory storage processes. In W. H. Gispen, T. B. van Wimersma Greidanus, B. Bohus, \& D. de Wied (Eds.), Progress in brain research (Vol. 42) Hormones, homeostasis and the brain. Amsterdam: Elsevier Scientific, 1975.

Miller, R. R., \& Springer, A. D. Amnesia, consolidation and retrieval. Psychological Review, 1973, 80, 69-79.

Rescorla, R. A., \& Heth, C. D. Reinstatement of fear to an extinguished conditioned stimulus. Journal of Experimental Psychology: Animal Behavior Processes, 1975, 104, 88-96.

Schuster, C. R., \& Brady, J. V. The discriminative control of a food reinforced operant by interoceptive stimulation. Pavlovian Journal of Higher Nervous Activity, 1964, 14, 448-458.

Spear, N. E., \& Parsons, P. Analysis of a reactivation treatment: Ontogeny and alleviated forgetting. In D. Medin, R. Davis, \& W. Roberts (Eds.), Processes of animal memory. Hillsdale, N.J: Erlbaum, 1976.

(Received for publication February 6, 1979; revision accepted August 7, 1979.) 